\title{
Rate Analysis of Uplink Massive MIMO with Low-Resolution ADCs and ZF Detectors over Rician Fading Channels
}

\author{
Author A, Author B, Author C, and Author D
}

\begin{abstract}
In order to increase energy efficiency (EE), analogto-digital converters (ADC) with a low resolution are used to reduce the internal power consumption in massive multiple-input multiple-output (MIMO) systems. In this work, we derive the uplink spectral efficiency (SE) of massive MIMO systems with low-resolution ADCs over Rician fading channels, and using zeroforcing (ZF) detectors. To obtain a closed-form expression of the sum-rate, we adopt the additive quantization noise model (AQNM), where the quantization noise is treated as an additive and independent noise. Our analysis is supported by various numerical results. For instance, compared to Rayleigh fading, specific conditions under Rician fading are needed to keep a good tradeoff between $\mathrm{EE}$ and system performance while using low-resolution ADCs.
\end{abstract}

Index Terms-Achievable Rate, ADC, AQNM, Massive MIMO, Rician Fading, Spectral Efficiency, ZF.

\section{INTRODUCTION}

$\mathbf{M}$ ASSIVE multiple input multiple output (MIMO) is one of the promising technologies for future wireless networks, using $M$ antennas at the base station (BS), and serving simultaneously $N$ users, can achieve clear improvements in terms of capacity and link reliability [1] [2]. In particular, it has been shown that the transmit power is reduced by $1 / M$ when perfect channel state information (CSI) is available.

On the other hand, energy efficiency (EE) has become a key metric in emerging technologies. Many research works are exploring ways to keep increasing the energy gains in massive MIMO. One main internal block that consumes considerable energy is the analog-to-digital converter (ADC), where the power consumption scales exponentially with the number of resolution bits [3]. As a result, the use of a large number of antennas, and thus of ADCs, leads to higher energy consumption. In response, low-resolution ADCs are proposed to increase EE [4]-[8].

The effect of low-resolution ADCs on the uplink achievable rate under Rayleigh fading was investigated in [5] [6], using respectively maximum ratio combining (MRC) and zeroforcing (ZF) detection techniques. However, measurements have shown that a dominant propagation direction is mostly experienced [9]. Therefore, it is of much interest to consider the Rician fading model which is more realistic and fits a larger range of scenarios such as millimeter wave communications, vehicular-to-vehicular systems, and indoor communications [10], [11]. In the literature, only a few papers discussing low resolution ADCs have considered Rician fading [7], [8], where both papers have explored the uplink sum-rate using MRC detection receivers.
In this paper, we analyze the uplink achievable rate over Rician fading with low-resolution ADCs and using ZF detectors which has been proven to outperform MRC schemes. For more convenience, we are using the additive quantization noise model (AQNM) which is the most used model in the literature. Assuming perfect CSI at the BS side, we derive a closed-form expression for the achievable rate in uplink. In addition, we explore the parameters that affect the spectral efficiency (SE) and EE, including the Rician factor and the number of resolution bits. Our results are a generalization of previous works which assume ideal Rayleigh fading channels, or the basic MRC scheme [5]-[8].

Notations: $X^{*}, X^{\mathrm{H}}, \operatorname{tr}(X),\|X\|$, and $|X|$ denote, respectively, the conjugate, the conjugate transpose, the trace, the Euclidean norm, and the module of $X$. For an integer $n$, we denote by $n$ !! the semi-factorial operation, i.e., $n ! !=$ $n \cdot(n-2) \cdots 2$ if $n$ is even, and $n ! !=n \cdot(n-2) \cdots 1$ otherwise.

\section{SYSTEM MODEL}

We consider the uplink of a single-cell multi user massive MIMO system where $N$ single-antenna users transmit their signals to an $M$-antenna BS, sharing the same time-frequency resource. We assume that no interference is caused by users from adjacent cells [10] and that full knowledge of CSI is available. For a more complete analysis, the impact of imperfect CSI will be considered in a future extension of this work. We denote by $p_{\mathrm{t}}$ and $\mathbf{x}$ the transmitted power per user and the $N \times 1$ vector of symbols transmitted by the users. The $M \times 1$ signal vector $\mathbf{y}$ received at the BS side can be expressed as

$$
\mathbf{y}=\sqrt{p_{\mathrm{u}}} \mathbf{G x}+\mathbf{n},
$$

where $\mathbf{G}$ is denoting the $M \times N$ channel matrix between users and $\mathrm{BS}$, and $\mathbf{n}$ is the $M \times 1$ vector of zero-mean additive white Gaussian noise (AWGN) with unit variance.

\section{A. Rician Channel with Low-Resolution ADCs}

We consider that the channel matrix $\mathbf{G}$ models both independent small scale Rician fading and large scale fading [10],

$$
\mathbf{G}=\mathbf{H D}^{1 / 2}
$$

where $\mathbf{D}$ is a $N \times N$ diagonal matrix modeling large scale variations (path loss and shadowing) and assumed to be constant during the transmission. The small scale fading matrix 
$\mathbf{H}$ models the arbitrary rank Rician fading with a different factor $\mathrm{K}_{n}$ for each user [11]

$$
\mathbf{H}=\mathbf{H}_{\mathrm{L}}\left[\boldsymbol{\Omega}\left(\boldsymbol{\Omega}+\mathbf{I}_{N}\right)^{-1}\right]^{1 / 2}+\overline{\mathbf{H}}\left[\left(\boldsymbol{\Omega}+\mathbf{I}_{N}\right)^{-1}\right]^{1 / 2},
$$

with $\Omega$ a $N \times N$ diagonal matrix with $[\Omega]_{n n}=\mathrm{K}_{n}$, and $\left[\mathbf{H}_{\mathrm{L}}\right]_{m n} \triangleq \sigma_{m n}=\mathrm{e}^{-j(m-1) \pi \sin \left(\Theta_{n}\right)}$, where $\Theta_{n}$ is the arrival angle of the $n$-th user signal. Matrix $\overline{\mathbf{H}}$ is the random component whose entries are independent and identically distributed Gaussian random variables with zero-mean. The real and imaginary parts of each entry $[\overline{\mathbf{H}}]_{m n} \triangleq q_{m n}=s_{m n}+j t_{m n}$ are independent and each with variance $1 / 2$. Since the quantization error can be approximated as a linear gain with AQNM, the output vector of low-resolution ADCs can be expressed as [6]

$$
\mathbf{y}_{\mathrm{q}}=\mathbb{Q}\{\mathbf{y}\}=\alpha \mathbf{y}+\mathbf{n}_{\mathrm{q}}=\alpha \sqrt{p_{\mathrm{u}}} \mathbf{G} \mathbf{x}+\alpha \mathbf{n}+\mathbf{n}_{\mathrm{q}},
$$

$\alpha=1-\rho$ being the distortion factor where $\rho=\sqrt{3} \pi 2^{-2 b-1}$ when $b>5$; and for $b \leq 5$ the exact values are given by [5]. The additive Gaussian quantization noise vector $\mathbf{n}_{\mathrm{q}}$ is uncorrelated with $\mathbf{y}$. For a fixed channel realization $\mathbf{G}$, the covariance matrix of $\mathbf{n}_{\mathrm{q}}$ can be expressed as [6]

$$
\mathbf{R}_{\mathbf{n}_{\mathrm{q}} \mathbf{n}_{\mathrm{q}}}=\alpha(1-\alpha) \operatorname{diag}\left(p_{\mathrm{u}} \mathbf{G G}^{\mathrm{H}}+\mathbf{I}_{N}\right) .
$$

\section{B. Uplink Achievable Rate}

We denote by $\mathbf{A}$ the linear detection matrix, and by $\mathbf{a}_{n}$ and $\mathbf{g}_{n}$ the $n$-th columns of $\mathbf{A}$ and $\mathbf{G}$, respectively. By multiplying $\mathbf{y}_{\mathrm{q}}$ with $\mathbf{A}^{\mathrm{H}}$, the uplink achievable rate can be expressed as

$$
R_{n}=\mathbb{E}\left\{\log _{2}\left(1+\frac{\alpha^{2} p_{\mathrm{u}}\left|\mathbf{a}_{n}^{\mathrm{H}} \mathbf{g}_{n}\right|^{2}}{\Psi_{\mathbf{G}}}\right)\right\}
$$

where $\Psi_{\mathbf{G}}=\alpha p_{\mathrm{u}} \sum_{i \neq n}^{N}\left|\mathbf{a}_{n}^{\mathrm{H}} \mathbf{g}_{i}\right|^{2}+\alpha\left\|\mathbf{a}_{n}\right\|^{2}+$ $\alpha(1-\alpha) \mathbf{a}_{n}^{\mathrm{H}} \operatorname{diag}\left(\mathbf{G G}^{\mathrm{H}}+\mathbf{I}_{N}\right) \mathbf{a}_{n}$. In the remainder, we define the uplink sum rate of the cell as the total sum of the $N$ users' achievable rates.

\section{SUM-RATE ANALYSIS}

Adopting ZF, the detection matrix can be expressed as $\mathbf{A}=$ $\mathbf{G}\left(\mathbf{G}^{\mathrm{H}} \mathbf{G}\right)^{-1}$. The uplink rate is hence given by [6]

$R_{n}=\mathbb{E}\left\{\log _{2}\left(1+\frac{\alpha p_{\mathrm{u}}}{\alpha\left\|\mathbf{a}_{n}\right\|^{2}+(1-\alpha) \mathbf{a}_{n}^{\mathrm{H}} \operatorname{diag}\left(\mathbf{G G}^{\mathrm{H}}+\mathbf{I}_{N}\right) \mathbf{a}_{n}}\right)\right\}$.

To compute (7), we need to derive the expectations of the three first absolute moments of the channel coefficients $\mathbf{g}_{m n}$. The first and second moments are found with the help of [11, Lemma 3] as

$$
\mathbb{E}\left\{\left|g_{m n}\right|^{2}\right\} \triangleq \beta_{n}=[\mathbf{D}]_{n n}
$$

and

$$
\mathbb{E}\left\{\left|g_{m n}\right|^{4}\right\} \triangleq \gamma_{n}=\beta_{n}^{2}\left(M+\frac{2 \mathrm{~K}_{n}+1}{\left(\mathrm{~K}_{n}+1\right)^{2}}\right),
$$

Lemma 1. The expectation of the third absolute moments of the channel coefficients $g_{m n}$ is given by

$$
\begin{aligned}
\mathbb{E}\left\{\left|g_{m n}\right|^{6}\right\} & \triangleq \lambda_{n} \\
& =\beta_{n}^{3}\left(M^{2}+\frac{M\left(6 \mathrm{~K}_{n}^{2}+9 \mathrm{~K}_{n}+3 / 2\right)+5 / 4}{\left(\mathrm{~K}_{n}+1\right)^{3}}\right) .
\end{aligned}
$$

Proof. Noting that the entries of $\mathbf{H}$ can be expressed as

$$
[\mathbf{H}]_{m n}=\sqrt{\frac{\mathrm{K}_{n}}{\mathrm{~K}_{n}+1}} \sigma_{m n}+\sqrt{\frac{1}{\mathrm{~K}_{n}+1}} q_{m n},
$$

the product $\mathbf{h}_{n}^{\mathrm{H}} \mathbf{h}_{n}$ is expressed, as in [11], under the form

$$
\begin{aligned}
\mathbf{h}_{n}^{\mathrm{H}} \mathbf{h}_{n}= & \sum_{m=1}^{M}\left\{\frac{\mathrm{K}_{n}}{\mathrm{~K}_{n}+1}+\frac{\sqrt{\mathrm{K}_{n}}}{\mathrm{~K}_{n}+1} \sigma_{m n}^{*} q_{m n}\right. \\
& \left.+\frac{\sqrt{\mathrm{K}_{n}}}{\mathrm{~K}_{n}+1} \sigma_{m n} q_{m n}^{*}+\frac{1}{\mathrm{~K}_{n}+1}\left|q_{m n}\right|^{2}\right\} .
\end{aligned}
$$

Note that $\mathbf{h}_{n}^{\mathrm{H}} \mathbf{h}_{n}$ includes only a real part. Thus, we can derive the norm-cube of the vector $\mathbf{g}_{n}$ as in (13) (depicted on the top of the next page) with $\rho_{m n} \triangleq s_{m n} \operatorname{Re}\left(\left[\mathbf{H}_{\mathrm{L}}\right]_{m n}\right)-$ $t_{m n} \operatorname{Im}\left(\left[\mathbf{H}_{\mathrm{L}}\right]_{m n}\right), \mu_{m n} \triangleq\left|q_{m n}\right|^{2}, \varrho_{n} \triangleq \sum_{m=1}^{M} \rho_{m n}$, and $\nu_{n} \triangleq \sum_{m=1}^{M} \mu_{m n}$.

Furthermore, it is easy to show the following approximation for independent variables $X_{i}$

$$
\begin{array}{r}
\mathbb{E}\left\{\left(\sum_{i=1}^{M} X_{i}\right)^{3}\right\}=M \mathbb{E}\left(X_{i}^{3}\right)+3 M(M-1) \mathbb{E}\left(X_{i}^{2}\right) \\
+\mathbb{E}\left(X_{i}\right)+M(M-1)(M-2) \mathbb{E}\left(X_{i}\right)^{3} .
\end{array}
$$

This approximation yields closed-form expressions for the terms $\mathbb{E}\left\{\varrho_{n}^{3}\right\}$ and $\mathbb{E}\left\{\nu_{n}^{3}\right\}$. Since both $s_{m n}$ and $t_{m n}$ follow normal distributions with the same variance $\sigma^{2}=1 / 2$, for any non-negative integer $k$, their plain central moments can be expressed as $\mathbb{E}\left\{s_{m n}^{k}\right\}=\mathbb{E}\left\{t_{m n}^{k}\right\}=\sigma^{k}(k-1)$ !! when $k$ is even, and $\mathbb{E}\left\{s_{m n}^{k}\right\}=\mathbb{E}\left\{t_{m n}^{k}\right\}=0$ otherwise. In addition, considering that $\mathbb{E}\left\{s_{m n}^{4}\right\}=\mathbb{E}\left\{t_{m n}^{4}\right\}=3 / 4$, and $\mathbb{E}\left\{s_{m n}^{6}\right\}=\mathbb{E}\left\{t_{m n}^{6}\right\}=15 / 8$, the final result is obtained after a few strainghtforward simplifications.

Theorem 1. For an uplink multi-user massive MIMO system with low-resolution ADCs ZF detectors, the achievable sumrate of the $n$-th user can be approximated by

$R_{n}=\mathbb{E}\left\{\log _{2}\left(1+\frac{\alpha p_{\mathrm{u}}}{\left.\frac{\alpha}{\beta_{n}(M-N)}\left[\hat{\sum}^{-1}\right]_{n n}+(1-\alpha) M L^{2} \mathbb{I}_{G}\right)}\right)\right\}$,

where $\mathbb{I}_{G}$ is depicted in (20) (on top of next page), with $L \triangleq$ $\delta /(M+N)$ and $\delta<1$ is the attenuation parameter [6]. Matrix $\hat{\mathbf{\Sigma}}$ is defined as

$$
\begin{aligned}
\hat{\boldsymbol{\Sigma}} & =\left(\boldsymbol{\Omega}+\mathbf{I}_{\mathrm{K}}\right)^{-1} \\
& +\frac{1}{M}\left[\boldsymbol{\Omega}\left(\boldsymbol{\Omega}+\mathbf{I}_{\mathrm{K}}\right)^{-1}\right]^{1 / 2} \cdot \mathbf{H}_{L}^{\mathrm{H}} \mathbf{H}_{L} \cdot\left[\boldsymbol{\Omega}\left(\boldsymbol{\Omega}+\mathbf{I}_{\mathrm{K}}\right)^{-1}\right]^{1 / 2} .
\end{aligned}
$$

while the following lemma gives the third moment. 


$$
\begin{aligned}
\left\|\mathbf{g}_{n}\right\|^{6}= & \left(\frac{M \mathrm{~K}_{n}}{\mathrm{~K}_{n}+1}\right)^{3}+\frac{8 \mathrm{~K}_{n} \sqrt{\mathrm{K}_{n}}}{\left(\mathrm{~K}_{n}+1\right)^{3}} \varrho_{n}^{3}+\frac{3 M \mathrm{~K}_{n}}{\left(\mathrm{~K}_{n}+1\right)^{3}} \nu_{n}^{2}+\frac{1}{\left(\mathrm{~K}_{n}+1\right)^{3}} \nu_{n}^{3}+\frac{3 M^{2} \mathrm{~K}_{n}^{2}}{\left(\mathrm{~K}_{n}+1\right)^{3}} \nu_{n}+\frac{6 M^{2} \mathrm{~K}_{n}^{2} \sqrt{\mathrm{K}_{n}}}{\left(\mathrm{~K}_{n}+1\right)^{3}} \varrho_{n}+\frac{6 \sqrt{\mathrm{K}_{n}}}{\left(\mathrm{~K}_{n}+1\right)^{3}} \varrho_{n} \cdot \nu_{n}^{2} \\
& +\frac{12 M \mathrm{~K}_{n} \sqrt{\mathrm{K}_{n}}}{\left(\mathrm{~K}_{n}+1\right)^{3}}\left(\sum_{m_{1}=1}^{M} \sum_{m_{2}}^{M} \rho_{m_{1} n} \cdot \mu_{m_{2} n}\right)+\frac{12 M \mathrm{~K}_{n}^{2}}{\left(\mathrm{~K}_{n}+1\right)^{3}} \varrho_{n}^{2}+\frac{12 \mathrm{~K}_{n}}{\left(\mathrm{~K}_{n}+1\right)^{3}} \varrho_{n}^{2} \nu_{n}
\end{aligned}
$$

$$
\begin{aligned}
& E_{1}=4 \beta_{n}-4 L \gamma_{n}+M L^{2} \beta_{n} \gamma_{n}-4 L \beta_{n} \sum_{\substack{j=1, j \neq n}}^{N} \beta_{j}+M L^{2} \beta_{n} \sum_{\substack{j=1, j \neq n}}^{N} \beta_{j}^{2} \\
& E_{2}=4 \beta_{n} \beta_{h}-4 L\left(\beta_{h} \gamma_{n}+\beta_{n} \gamma_{h}\right)+L^{2} \beta_{n} \beta_{h}\left(\gamma_{n}+\gamma_{h}\right)+4 L \beta_{n} \beta_{h} \sum_{\substack{j=1 \\
j \neq n, h}}^{N} \beta_{j}+L^{2} \beta_{n} \beta_{h} \sum_{\substack{j=1 \\
j \neq n, h}}^{N} \beta_{j}^{2} \\
& E_{3}=4 \gamma_{n}-4 L \lambda_{n}+\beta_{n} \lambda_{n} L^{2}+\gamma_{n}^{2} L^{2}(M-1)-4 L \gamma_{n} \sum_{\substack{j=1 \\
j \neq n}}^{N} \beta_{j}+\gamma_{n} L^{2} \sum_{\substack{j=1 \\
j \neq n}}^{N} \beta_{j}^{2}+\beta_{n}^{2} L^{2}(M-1) \sum_{\substack{j=1 \\
j \neq n}}^{N} \beta_{j}^{2}
\end{aligned}
$$

$$
\begin{aligned}
\mathbb{I}_{G}= & 4 \beta_{n}-4 L\left(\beta_{n} \sum_{\substack{j=1 \\
j \neq n}}^{N} \beta_{j}+\gamma_{n}\right)+M L^{2}\left(\beta_{n} \sum_{\substack{j=1 \\
j \neq n}}^{N} \beta_{j}^{2}+\beta_{n} \gamma_{n}\right) \\
& +p_{\mathrm{u}} \sum_{\substack{h=1 \\
h \neq n}}^{N}\left[4 \beta_{n} \beta_{h}-4 L\left(\beta_{h} \gamma_{n}+\beta_{n} \gamma_{h}+\beta_{n} \beta_{h} \sum_{\substack{j=1 \\
j \neq n, h}}^{N} \beta_{j}\right)+L^{2} \beta_{n} \beta_{h}\left(\gamma_{n}+\gamma_{h}+\sum_{\substack{j=1 \\
j \neq n, h}}^{N} \beta_{j}^{2}\right)\right] \\
& +p_{\mathrm{u}}\left[4 \gamma_{n}-4 L\left(\lambda_{n}+\gamma_{n} \sum_{\substack{j=1 \\
j \neq n}}^{N} \beta_{j}\right)+L^{2}\left(\beta_{n} \lambda_{n}+\gamma_{n} \sum_{\substack{j=1 \\
j \neq n}}^{N} \beta_{j}^{2}\right)+L^{2}(M-1)\left(\gamma_{n}^{2}+\beta_{n}^{2} \sum_{\substack{j=1 \\
j \neq n}}^{N} \beta_{j}^{2}\right)\right]
\end{aligned}
$$

Proof. Based on the fact that the Gramm matrix $\mathbf{G}^{\mathrm{H}} \mathbf{G}$ follows a non-central Wishart distribution, the first term in the denominator of (7) can be expressed as in [10] as $\mathbb{E}\left\{\left\|\mathbf{a}_{n}\right\|^{2}\right\}=$ $\left(\beta_{n}(M-N)\right)^{-1}\left[\hat{\Sigma}^{-1}\right]_{n n}$. We denote by $\mathbb{I}_{G}$ the second term in the denominator of (7) which is more complicated to derive, and we leverage the polynomial expansion which provides an accurate approximation [6]

$$
\begin{aligned}
M L^{2} \mathbb{I}_{G}= & \mathbb{E}\left\{\mathbf{a}_{n}^{\mathrm{H}} \operatorname{diag}\left(\mathbf{G G}^{\mathrm{H}}+\mathbf{I}_{N}\right) \mathbf{a}_{n}\right\} \\
= & \sum_{m=1}^{M} \mathbb{E}\left\{\frac{\left|a_{m n}\right|^{2}}{L^{2}}\right\} \\
& +p_{\mathrm{u}} \sum_{m=1}^{M} \mathbb{E}\left\{\frac{\left|a_{m n}\right|^{2}}{L^{2}} \cdot \sum_{h=1, h \neq n}^{N}\left|g_{m h}\right|^{2}\right\} \\
& +p_{\mathrm{u}} \sum_{m=1}^{M} \mathbb{E}\left\{\frac{\left|a_{m n}\right|^{2}}{L^{2}} \cdot\left|g_{m n}\right|^{2}\right\} .
\end{aligned}
$$

The first, second and third terms of the right hand side of (21), denoted respectively by $E_{1}, p_{\mathrm{u}} E_{2}, p_{\mathrm{u}} E_{3}$, are calculated separately as shown in (17), (18), and (19) (above). Substituting these three expressions in (21), we obtain the expression of $\mathbb{I}_{G}$ as in (20).

\section{Numerical Results ANd Discussion}

We consider a massive MIMO multi-user single-cell of radius $r_{\max }=1000$ meters. The $N$ users are distributed in a random but uniform way throughout the cell, with the exclusion of a central disk of radius $r_{\min }=50$ meters. The large-scale variations $\mathbf{D}$ is modeled by $\beta_{n}=z_{n} /\left(\mathbf{r}(n) / r_{\min }\right)^{v}$, where $\mathbf{r}$ is a $N \times 1$ vector whose $n$-th element is the distance from user $n$ to the $\mathrm{BS}, z_{n}$ is a log-normal random variable with a standard deviation of $8 \mathrm{~dB}$, and $v=3.8$ is the decay exponent. We assume that the angles $\Theta_{n}$ are uniformly distributed in $[-\pi / 2, \pi / 2]$. Throughout the simulations, we consider a common Rician factor $\mathrm{K}=\mathrm{K}_{1}=\ldots=\mathrm{K}_{N}$ for all users.

In Fig. 1(a), we first validate the accuracy of our proposed approximation in Theorem 1, considering different ADC resolutions $b=2,6, \infty$ bits.

In Fig. 1(b) we illustrate the role of the Rician K-factor on the SE performance for a fixed ADC resolution $b=2 ; 3 ; 4 ; 6$. We can notice that performance loss due to low-resolution ADCs becomes more important with the increase of Rician Kfactor. This means that the low-resolution has a more negative impact over Rician fading than over Rayleigh channels (i.e., $K=1$ ). This might be justified by the channel fading nature 


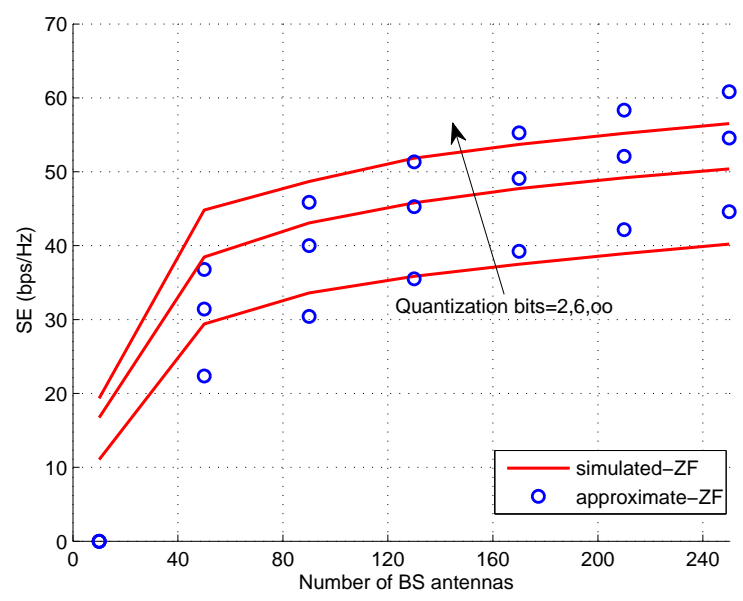

(a)

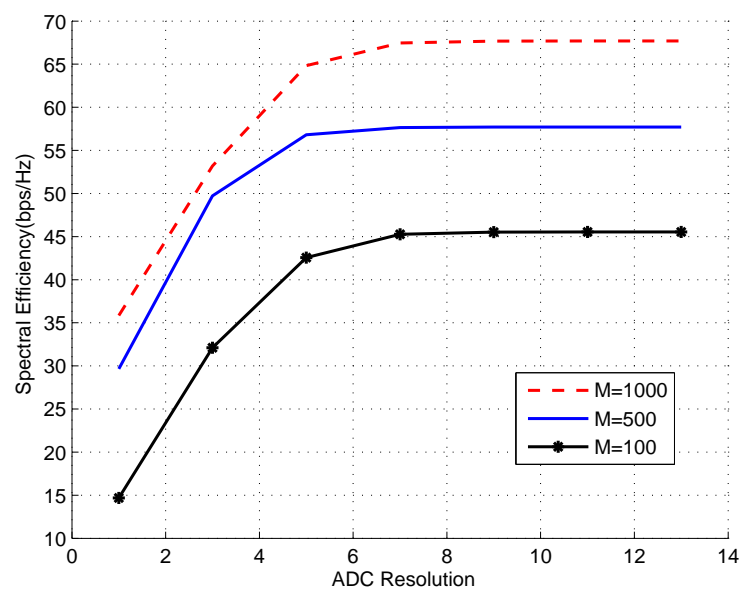

(c)

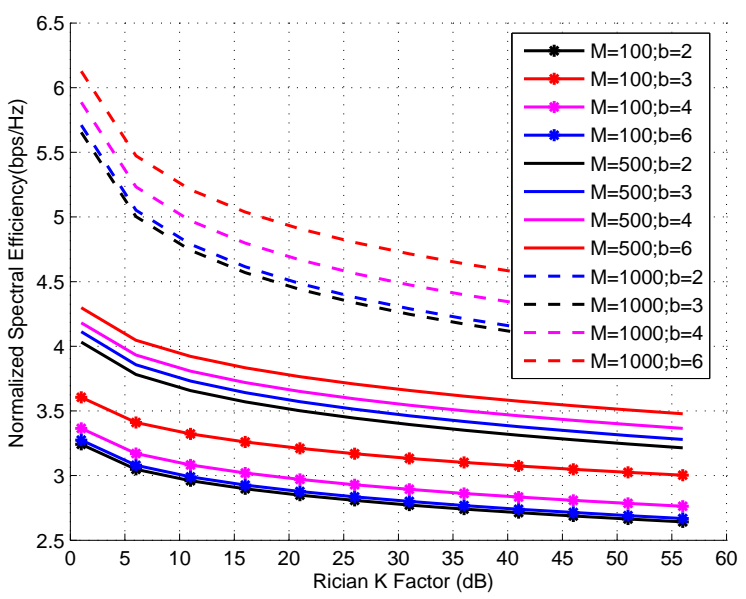

(b)

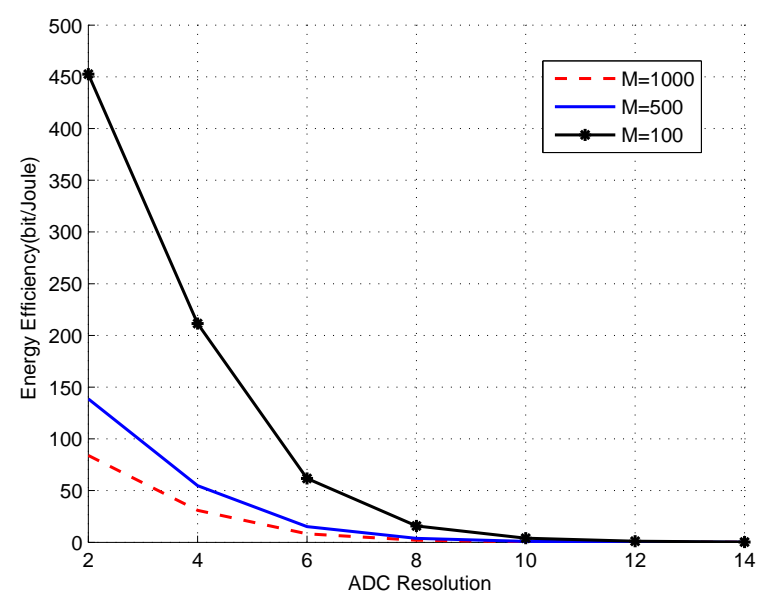

(d)

Fig. 1. (a) Simulated and analytical achievable SE versus the number of BS antennas, for $N=10, p_{\mathrm{u}}=10 \mathrm{~dB}$, and $\mathrm{K}=10 \mathrm{~dB}$, (b) normalized sum-rate versus $\mathrm{K}$ for $N=10, p_{\mathrm{u}}=10 \mathrm{~dB}$, and $\mathrm{ADC}$ resolution $b=2 ; 3 ; 4 ; 6$ bits, (c) spectral efficiency; and (d) energy efficiency versus the number of ADC resolution bits, for $\mathrm{K}=10 \mathrm{~dB}, N=10$, and $p_{\mathrm{u}}=10 \mathrm{~dB}$.

that becomes more deterministic than random. We note that our results remain accurate even for Rician K-factors as high as $20 \mathrm{~dB}$.

Finally, in Fig. 1(c) and Fig. 1(d), in addition to the SE, EE is also illustrated as the other key performance metric defined as $\eta=B R / P$ with $P=c_{0} 2^{b} M+c_{1}$, where $c_{0}=10^{-4} \mathrm{~W}$ and $c_{1}=0.02 \mathrm{~W}$, and $B$ is the bandwidth set here to $1 \mathrm{MHz}$. As a result, and for different antenna numbers $M=100,500$, and 1000 , we note that the intermediate values of ADC resolution between $b=5$ and $b=7$ bits provide a good SE-EE tradeoff.

Low-resolution ADCs are a potential approach to increase the EE of practical massive MIMO systems. As a future extension, it is worth to explore the impact of imperfect CSI on the obtained results to have a better understanding of the behavior of the analyzed scheme in more realistic conditions.

\section{REFERENCES}

[1] E. G. Larsson, O. Edfors, F. Tufvesson, and T. L. Marzetta, "Massive MIMO for next generation wireless systems", in IEEE Communications Magazine, vol. 52, no. 2, pp. 186-195, Feb. 2014.
[2] J. Hoydis, S. ten Brink, and M. Debbah, "Massive MIMO in the UL/DL of cellular networks: How many antennas do we need?", in IEEE Journal on Selected Areas in Communications, vol. 31, no. 2, pp. 160-171, Feb. 2013.

[3] R. H. Walden, "Analog-to-digital converter survey and analysis", in IEEE Journal on Selected Areas in Communications, vol. 17, no. 4, pp. 539550, Apr. 1999.

[4] C. Risi, D. Persson, and E. G. Larsson, "Massive MIMO with 1-bit ADC", arXiv:1404.7736, 2014

[5] L. Fan, S. Jin, C. K. Wen, and H. Zhang, "Uplink achievable rate for massive MIMO systems with low-resolution ADC", in IEEE Communications Letters, vol. 19, no. 12, pp. 2186-2189, Dec. 2015.

[6] D. Qiao, W. Tan, Y. Zhao, C. K. Wen, and S. Jin, "Spectral efficiency for massive MIMO zero-forcing receiver with low-resolution ADC", in Proc. 8th International Conference on Wireless Communications and Signal Processing (WCSP), Yangzhou, China, Oct. 2016.

[7] J. Zhang, L. Dai, S. Sun, and Z. Wang, "On the Spectral Efficiency of Massive MIMO Systems With Low-Resolution ADCs", in IEEE Communications Letters, vol. 20, no. 5, pp. 842-845, May 2016.

[8] J. Zhang, L. Dai, Z. He, S. Jin, and X. Li, "Performance Analysis of Mixed-ADC Massive MIMO Systems over Rician Fading Channels", in IEEE Journal on Selected Areas in Communications, vol. 35, no. 6, pp. 1327-1338, Jun. 2017.

[9] S. Gunnarsson, J. Flordelis, L. Van der Perre, and F. Tufvesson, "Channel Hardening in Massive MIMO-A Measurement Based Analysis", in Proc. IEEE 19th International Workshop on Signal Processing Advances in Wireless Communications (SPAWC), Kalamata, Greece, Jun. 2018. 
[10] S. Ghacham, M. Benjillali, and Z. Guennoun, "Low-complexity detection for massive MIMO systems over correlated Rician fading", in Proc. 13th International Wireless Communications and Mobile Computing Conference (IWCMC), pp. 1677-1682, Valencia, Spain, Jun. 2017.
[11] Q. Zhang, S. Jin, K. K. Wong, H. Zhu, and M. Matthaiou, "Power scaling of uplink massive MIMO systems with arbitrary-rank channel means", in IEEE Journal of Selected Topics in Signal Processing, vol. 8, no. 5, pp. 966-981, Oct. 2014. 